\title{
Ground Data System Risk Mitigation Techniques For Faster, Better, Cheaper Missions'
}

\author{
John Catena \\ NASA Goddard Space Flight Center \\ Greenbelt, MD 20771 \\ 301-286-9572 \\ john.catena@gsfc.nasa.gov \\ Rick Saylor \\ Honeywell Technical Solutions Inc. \\ Greenbelt, MD 2077I \\ 301-286-1354 \\ rick.saylor@honeywell-tsi.com
}

\author{
Ralph Casasanta \\ Computer Sciences Corporation \\ Lanham, MD 20706 \\ 301-794-2076 \\ rcasasan@csc.com \\ Craig Weikel \\ Computer Sciences Corporation \\ Lanham, MD 20706 \\ 301-794-2078 \\ cweikel@csc.com
}

\begin{abstract}
With the advent of faster, cheaper and better missions, NASA Projects acknowledged that a higher level of risk was inherent and accepted with this approach. It was incumbent however upon each component of the Project whether spacecraft, payload, launch vehicle or ground data system to ensure that the mission would nevertheless be an unqualified success.
\end{abstract}

The Small Explorer (SMEX) program's ground data system (GDS) team developed risk mitigation techniques to achieve these goals starting in 1989. These techniques have evolved through the SMEX series of missions and are practiced today under the Triana program. These techniques are:

1. Mission Team Organization - empowerment of a closeknit ground data system team comprising system engineering, software engineering, testing, and flight operations personnel,

2. Common Spacecraft Test \& Operational Control System - utilization of the pre-launch spacecraft integration system as the post-launch ground data system on-orbit command and control system,

3. Utilization of operations personnel in pre-launch testing - making the flight operations team an integrated member of the spacecraft testing activities at the beginning of the spacecraft fabrication phase,

4. Consolidated Test Team - combined system, mission readiness and operations testing to optimize test opportunities with the ground system and spacecraft, and

5. Reuse of Spacecraft, Systems and People - reuse of people, software and on-orbit spacecraft throughout the SMEX mission series.

The SMEX ground system development approach for faster, cheaper, better missions has been very successful. This paper will discuss these risk management techniques in the areas of ground data system design, implementation, test and operational readiness.

\section{TABLE OF CONTENTS}

1. INTRODUCTION

2. THE SMEX ERA

3. Risk Mitigation TeChniques

4. CONCLUSIONS

5. REFERENCES

6. BIOGRAPHIES

\section{INTRODUCTION}

The normal practice for developing a GDS for a mission operated at the Goddard Space Flight Center (GSFC) in the 1980's utilized a development team and a flight operations team, both of which were organizationally independent of each other. In addition, they were independent of the Project team responsible for building and launching the satellite. All three groups interacted and coordinated common activities, but this interaction required close coordination, detailed documentation, frequent meetings and extensive testing.

The spacecraft architecture and design of hardware and software components varied from mission to mission with little commonality for the interfaces to the operational GDS.

Multi-mission institutional systems consisting of hundreds of thousands of lines of code were modified to accommodate the next mission. These modifications were expensive to implement and test. Retesting of existing operational missions was another burden on this approach. However, the state and cost of computer technology at this time made this the only viable approach.

${ }^{1}$ U.S. Government work not protected by U.S. copyright 
Prior to the advent of the faster, cheaper, better approach to NASA missions, the schedules for GDS implementation were nominally five years or more. In 1989 NASA identified a new Explorer-class program at GSFC that changed the way future missions and the associated GDS would be constructed, scheduled and budgeted. Now GDS development schedules were only three years in duration. The GDS had to drastically modify its approach to implementation and testing to support launch and on-orbit operations. The following discussion topics cover how we responded to these new challenges.

\section{THE SMEX ERA}

The SMEX Project charter was to provide frequent flight opportunities at three-year intervals, from NASA approval to launch. Higher risk to the mission, in exchange for these more frequent flight opportunities, was deemed acceptable.

The first missions selected for this new program were the Solar Anomalous and Magnetospheric Particle Explorer (SAMPEX), the Submillimeter Wave Astronomy Satellite (SWAS), and the Fast Auroral Snapshot Explorer (FAST). The original launch dates for these missions were SAMPEX in mid-1992, SWAS in mid-1993, and FAST in late 1993 [I].

In 1995, the next two missions were identified: the Transition Region and Coronal Explorer (TRACE) and the Wide-Field Infrared Explorer (WIRE). TRACE was scheduled for launch in 1998 and WIRE in 1999. Figure 1 depicts the original implementation schedule and operational life of each of these missions.

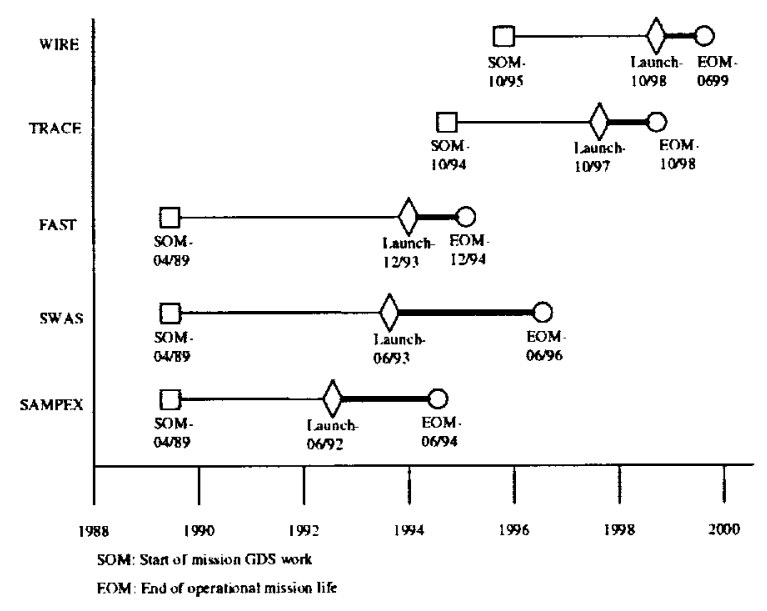

Figure 1. SMEX Mission Set $1 \& 2$ Schedules

Meeting the challenge of concurrent, compact mission schedules required innovations in GDS development and operational preparedness. However, not all these risk mitigation approaches were applied on day one. These approaches evolved over time as the GDS team gained experience and took advantage of changes internally and externally.

The SMEX Project, coupled with advances in computer workstation technology, provided GSFC with a new opportunity to build ground systems faster, while being more reliable and less expensive. Given the charter of frequent flight opportunities and capped costs, there was no choice. Although higher risks were deemed acceptable, mission success remained paramount and delays in completing the GDS were not permitted to impact the spacecraft launch schedule.

Two important advantages to SMEX GDS development activities occurred outside the control of the GDS organization team. These were the reuse of the spacecraft architecture and the proximity of the spacecraft vendor to the GDS team.

\section{Similar spacecraft architectures}

The first five spacecraft were designed and constructed by the same GSFC organization and followed similar implementation standards. The telemetry and command protocols used onboard the satellites adopted the Consultative Committee for Space Data Systems (CCSDS) standard. As a result interfaces were very similar between each GDS, more so than with previous missions.

\section{Location of spacecraft and GDS development activities}

Typically GSFC projects used a private contractor to build the spacecraft offsite and then deliver it to GSFC for several months of integration testing. Upon completion of integration testing, the spacecraft was shipped to the launch site. Under the SMEX program, the spacecraft was constructed on the GSFC campus making technical interaction between the two groups an almost daily occurrence over a two to three year period.

\section{Risk Mitigation TECHNIQUES}

In addition to the spacecraft architecture and location aspects of the SMEX Project, there were several GDS-based initiatives that improved the chances to meet the SMEX goals of cost and schedule while not compromising mission success.

These initiatives or risk mitigation techniques were: 1). empowerment of a close-knit ground data system team comprising system engineering, software engineering, testing, and flight operations personnel, 2). utilization of the pre-launch spacecraft integration and test (I\&T) system as the post-launch ground data system on-orbit command and control system, 3). utilization of flight operations personnel in pre-launch spacecraft testing activities, 4). combined system, mission readiness and operations testing to optimize test opportunities with the ground system and spacecraft, and $5)$. 
reuse of people, software and on-orbit spacecraft throughout the SMEX and Triana mission series.

This section will discuss these initiatives in terms of their ability to mitigate risks in the GDS in an environment of faster, cheaper and better missions.

\section{Mission Team Organization}

The SMEX GDS mission team, comprised of both contractors and civil servants, was responsible for the implementation and on-orbit operation of the GDS. The team was led by the Ground System Project Manager (GSPM). The GSPM was responsible for both the development of the GDS and its operation for the first 30 days of the on-orbit mission phase. Under the GSPM, the Implementation Manager (IM) was responsible for system engineering, software development and test efforts. Figure 2 presents the SMEX mission team organization.

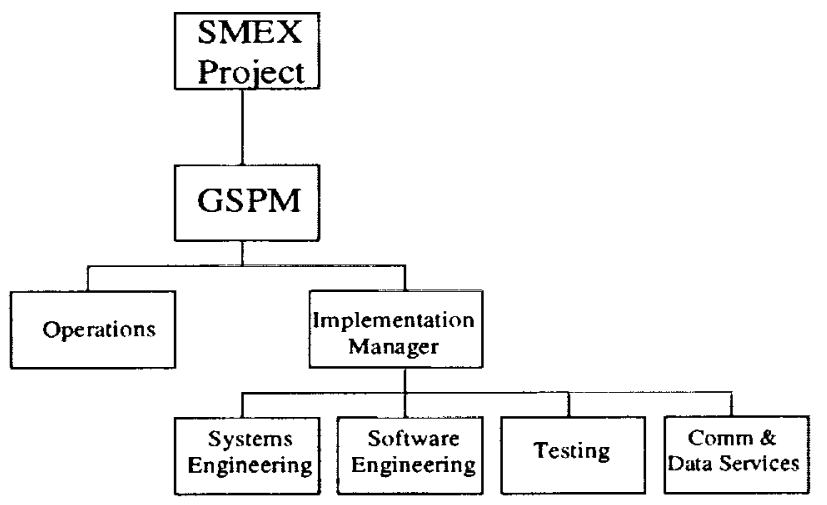

Figure 2. SMEX Mission Team Organization

The team developed GDS requirements as a unit, so the implementation was as efficient and cost effective as possible. Each member of the team was empowered to represent his or her discipline, regardless of "badge". They were each accountable for their deliveries, action items, and test schedule. They were encouraged to take ownership of the entire mission, with each part contributing to the success of the end-to-end system. The creed was "it is our mission". This mission team also was its' own configuration control board. Each potential change to the implementation of a particular set of requirements would have to pass a rigorous audit by the other members of the team, since each element change may impact another element within the data system. The IM would make the final decision with team input.

When identifying the many ways to implement a requirement, the mission team would discuss the risk factors of each option. Through trade studies or previous experience with that particular option, a plan would take shape. Then, this plan would be discussed amongst all involved on both the space and ground segments of the project, and the plan would be agreed to or altered accordingly. On occasion, there would be only one way to implement a requirement.
Then, rigorous testing would be the mitigating factor for risk analysis.

The team included ad-hoc members representing the science team, the spacecraft element, or the tracking services, which would participate on an infrequent basis depending on where the project was in the development cycle.

Other splinter working groups were formed as needed (i.e. RF working group, mission readiness testing group) to focus on particular issues or problems that affected a portion of the implementation team. These splinter groups would be assigned a chair, issue and track actions, and report back to the mission team where those actions would also be tracked until closed. Additionally, the GSPM would organize a Project wide retreat, where representatives from every element within the ground data system, spacecraft and instrument team would gather for 3-4 days. During this retreat, there would be presentations or tutorials to each other, informal requirement reviews, splinter working groups and the resolution of issues between elements. These workshops/retreats served as tremendous team building tools, while at the same time achieving significant results in closing issues sometimes 6 months earlier than normal.

Each mission team element lead engineer contributed significantly to the production of materials for presentation at major reviews, and while they may not actually stand and present; their input was sanctioned by the managers responsible for that particular discipline. By getting everyone on the team involved and assuming ownership and responsibility for the success of the entire system, you have synergy. It's not just the job of a single person, or the manager to apply risk management - it's everyone's responsibility.

At the conclusion of the launch phase of each mission, the mission team would re-convene and discuss lessons learned. What went right? What could have been done better? In a short, 3 -year development cycle, these lessons learned can be immediately applied to the next mission in the queue, although most likely, it's already underway.

\section{Common Spacecraft Test \& Operational Control System}

The Integrated Test and Operations System (ITOS) is a set of equipment that is used initially to interface with the spacecraft from the box level, all the way through to the observatory level. Along the way, the ITOS will add capabilities that the control center will utilize for command and control of the spacecraft when it is transferred to the Flight Operations Team on orbit. Using a common ground system for both I\&T and operations allows for an operations system to be launch capable much earlier than on previous missions [2]. The ITOS will have a factor of 10 more hours of test time with the spacecraft under this approach than on previous, operations-unique ground data systems. Also, combining the two systems bridges the gap between the 
space and ground segments on a typical mission. This is a tremendous team building technique.

By using a similar ground system during spacecraft I\&T and mission operations, problems are detected earlier. Earlier detection allows cleaner releases for the ground data system team. Another benefit for using the same ground system is that the development team is aware of mission requirements. The development team agrees to mission requirements and can apply this knowledge to program patches and enhancements.

There has always been a race to have all spacecraft procedures including contingencies tested prior to launch.

When SMEX had two different ground systems, most procedures were tested in the last month and large amounts of time had to be taken away from spacecraft I\&T testing to ensure that all procedures were verified against the actual spacecraft. Risk was reduced when SMEX decided to use a common system for spacecraft I\&T and operations because operation procedures would have more test time available.

Most of the procedures were developed for I\&T and reused in operations, and it reduced the need to dedicate spacecraft I\&T time for procedure testing for the operations team. The decision to use a common ground system enabled the operations team to play a larger role in the spacecraft $I \& T$ test and development areas.

\section{Utilization of Operations Team in Pre-Launch Testing}

In addition to leveraging the spacecraft I\&T system, for onorbit operations, the people responsible for operating the spacecraft on-orbit became key partners in the test and checkout of the spacecraft and payloads. It enabled the operations team to gain greater access to the spacecraft. This access is beneficial for performing testing and validating operations concepts. Greater access to the spacecraft transforms into knowledge and experience for the operations team reducing risk throughout the mission life.

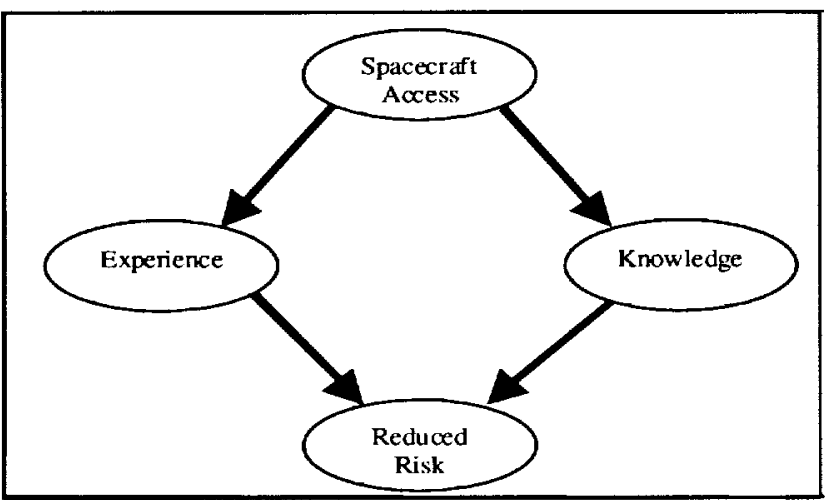

Figure 3. Spacecraft Access Benefit

Spacecraft access translated into greater spacecraft knowledge for the operations team. By performing testing during spacecraft I\&T, the operations team gained a better understanding of the spacecraft systems and instruments for the mission. Over time, the project looked at the test conductors as another set of system engineers monitoring the spacecraft development and testing. This gave the Project system engineers a higher confidence level during spacecraft I\&T and throughout the mission. A better trained and more knowledgable operations team reduced mission risk significantly during the operational phase of the mission. By participating in the spacecraft development and testing of the spacecraft, the operations team had the added knowledge of previous spacecraft anomalies found during I\&T. The operations team had better insight into which telemetry points to trend and monitor and it built a better relationship between the operations team and spacecraft engineers. The operations team had better access to the spacecraft engineers for the operational phase of the mission due to the working relationship developed during spacecraft I\&T.

The operations team gained experience by performing spacecraft I\&T testing. The operations team was tasked to stress test the spacecraft and ground system. This provided benefits to the development team and operations team, and reduced risk by minimizing the unknown perfromance characteristics of the spacecraft.

The experience gained during testing was transitioned to the operations phase of the mission since most of the team remained the same. The operations team was able to respond to anomalies quicker and were better prepared to handle anomaly situations. The seamless transition of the operations team from I\&T to on-orbit operations played a key role in anomaly investigations, as well as recovery operations.

Experience has enabled the operations team to become part of the spacecraft development team. By being part of the spacecraft development team, the operations team helped develop realistic operational testing scenarios, refine spacecraft design, and bridge the gap between the spacecraft and ground development teams. The operations team often assisted the system engineers in developing spacecraft test with operational scenarios. This allowed the spacecraft to be tested in the way that it would be operated on-orbit. As the operations team gained experience and knowledge, they helped refine spacecraft design by providing inputs and comments on flight software requirements and functions. These inputs were requested to help reduce the complexity of the operation concepts and with developing ground automation. For example, on the Triana mission, the C\&DH software group changed the stored command processor design from previous SMEX missions. The operations team played a key role in reviewing design requirements and testing. This not only helped the software developers but gave the operations team valuable insight into the functionality of the software. By including the operations team in development, overall operational risk was reduced.

Overall operational risk was also reduced because 
operational procedures and timelines were tested earlier, before major simulations and tests. This led to higher quality simulations and training for the entire project.

Other operational concepts were verified earlier such as trending. The operations team developed methods to test various ground components earlier. The result was increased test time for those components which would have otherwise been tested only during simulations and tests.

\section{Consolidated Test Team}

The ground system testing approach for the first three SMEX missions follow the traditional method used for most if not all GSFC ground systems in the 1980s. The development team delivered software to an independent system test team. This group was primarily responsible for requirements testing. Software anomalies or failures were documented using a problem reporting system specific to the system test group. Upon completion of system testing, the software element was delivered to a second independent test team that was responsible for acceptance testing. The primary responsibility of this group was to determine the ground system element's ability to support the operations environment. Another problem reporting system was used to track software anomalies detected in this phase. Upon completion of acceptance testing, the software was delivered to the operations environment. At this stage, the mission readiness manager and his team executed interface tests with all the ground system elements including the spacecraft, ground stations and the science team's home facilities. Again, a separate problem reporting system was used to track anomalies. In addition, each one of these groups had a separate test plan and procedures document with different test goals. The result of this testing approach was a thorough testing of the ground system but with a cost and schedule impact that was at odds with the faster, better, cheaper theme of SMEX missions.

With the selection of the second set of SMEX missions and launch delays for two of the original missions, a new process was needed to mitigate the risk of tighter schedules and overlapping mission activities. The new approach took advantage of the selection of ITOS as the operational command and control system and the larger amount of reuse enjoyed by the SMEX ground systems.

The new approach [3] adopted for the TRACE and WIRE missions consisted of the following

- unify the three test groups into one consolidated test team led by the mission readiness manager

- create a single test plan and procedures document which addressed the objectives of each of the three test groups

- make the most efficient use of tests by merging test objectives and eliminating redundancy

- consolidate the test reporting mechanism to one system used by all test members
- reduce the overall test group size but augmented the team with support from operations personnel

The results of the consolidated test approach for the TRACE mission when compared to the previous SMEX missions were significant. A 4-to-1 reduction in the test schedule was achieved while reducing the test team size by $50 \%$.

The ability of the consolidated test team to respond quickly and efficiently to ground system software patches and critical fixes became crucial in the later, hectic part of the SMEX Project at GSFC. The test team's rapid response to conflicting demands of the SMEX missions was a key factor in the successful support of three separate launches.

\section{Reuse of Spacecraft, Systems \& People}

Because each SMEX mission had a development cycle of approximately three-years from mission selection to launch, both the space-based and ground-based systems had to perform requirements development, system design, implementation, test and operations certification within this envelope. The quick access and rapid deployment resulted in modifications to the existing paradigms for both spaceand ground-based hardware fabrication and software development. The major emphasis was in the following areas:

- Common spacecraft architecture including similar or identical sensors, actuators and hardware components

- Reuse of flight- and ground-based software

- Better, more efficient use of personnel

\section{Common spacecraft architecture}

A major change to the spacecraft architecture was a common spacecraft bus used on the first SMEX missions and for the Triana mission. The Triana hardware architecture has a heritage from the earlier SMEX satellites and specifically the SMEX-Lite. This approach reduces spacecraft design and test engineering, development team size, flight hardware costs, and the cost of flight operations. This common bus approach also maintains mission reliability and improves performance over previous SMEX missions. The SMEXLite has "plug-and-play" architecture that allows components to be added or deleted from the system with virtually no redesign and without disturbing other subsystems. Functions are segregated into "slices" that are independent at the subsystem level. The "plug-and-play" concept was extended to the electronics, sensors, actuators, software, solar arrays, the mechanical system, and the ground support I\&T system.

\section{Reuse of flight-based and ground-based subsystems}

The Triana flight software enjoys a significant degree of heritage from previous SMEX missions. Its architecture utilizes modular design techniques that maximize software reuse. This approach provides flexibility for tailoring the 
system to unique mission requirements and improves the overall reliability of the flight code.

By using this standard set of hardware and software systems, the spacecraft development, fabrication, and certification time has been reduced to fit with the three-year lifecycle of mission selection to launch. Some upgrades were made to both the spacecraft hardware and software simply based on upgrading a component that was out-of-date.

An exceptionally important change for TRACE from previous SMEX missions was the merging of the I\&T system that was used for previous SMEX satellites with the independently developed mission operations system. In addition to the reduced software development costs, the new, combined ITOS system allowed display pages and procedures prepared by the FOT during the I\&T phase to also be used during the operational phase, resulting in great time and cost efficiencies for the FOT. The next SMEX mission, WIRE, used the same system for the I\&T and the operational phases. Also during this same time, the SWAS mission, which had its launch delayed until 1999, reengineered its GDS to use the same system. These three missions were launched and supported using the identical command and control system (ITOS) within eleven months of each other. As shown in Table 1, the SMEX software and hardware components of the GDS have been carried over into the Triana GDS. The one modification was the result of a spacecraft change related to how on-board commands are stored. Previously, the SMEX missions supported command buffers, which were used to store an absolute or relative time sequenced command or series of commands. With the advent of SMEX-Lite and the migration to the Triana mission, the on-board buffers were replaced with command tasks that are managed by the onboard command and data handling $(\mathrm{C} \& D H)$ subsystem. In this instance, the GDS minimized the risk by using the exact same command translator that is being developed by the spacecraft development team. The GDS did not develop its own separate system to perform the identical function.

Other GDS modifications for Triana are to support the updated telemetry and command mnemonics, as well as the file management concepts to support VxWorks (on-board and ground processed file management).

Additionally, the GDS is reusing to the extent possible any hardware systems that were used by the previous SMEX missions. When practical, the Triana program will purchase newer hardware components to upgrade to the latest hardware and operating systems or to purchase a faster, more powerful version of a similar hardware platform.

The SMEX missions and Triana are able to use the same suite of hardware to support a launch. By using this same

\begin{tabular}{|c|c|c|}
\hline $\begin{array}{c}\text { Ground Data } \\
\begin{array}{c}\text { System } \\
\text { Element }\end{array}\end{array}$ & $\begin{array}{c}\text { TRACE/WIRE } \\
\text { Software/Hardware } \\
\text { components }\end{array}$ & $\begin{array}{c}\text { Triana } \\
\text { Software/Hardware } \\
\text { components }\end{array}$ \\
\hline
\end{tabular}

\begin{tabular}{|l|l|l|}
\hline $\begin{array}{c}\text { Ground Data } \\
\text { System } \\
\text { Element }\end{array}$ & $\begin{array}{c}\text { TRACE/WIRE } \\
\text { Software/Hardware } \\
\text { components }\end{array}$ & $\begin{array}{l}\text { Triana } \\
\text { Software/Hardware } \\
\text { components }\end{array}$ \\
\hline $\begin{array}{l}\text { Command and } \\
\text { Control }\end{array}$ & $\begin{array}{l}\text { ITOS (SUN and PC } \\
\text { platforms) }\end{array}$ & $\begin{array}{l}\text { ITOS (SUN } \\
\text { platform) }\end{array}$ \\
\hline Trending & DTAS (PC) & DTAS (PC) \\
\hline $\begin{array}{l}\text { Attitude } \\
\text { Verification }\end{array}$ & ADS (PC) & ADS (PC) \\
\hline $\begin{array}{l}\text { Real-time } \\
\text { Attitude } \\
\text { Determination }\end{array}$ & RTADS (PC) & RTADS (PC) \\
\hline $\begin{array}{l}\text { Command } \\
\text { Management }\end{array}$ & MPS (SUN) & $\begin{array}{l}\text { Command } \\
\text { Translator (SUN) }\end{array}$ \\
\hline
\end{tabular}

Table 1. Comparison of GDS elements between earlier SMEX missions and Triana

set of hardware, the missions have eliminated risks associated with moving to completely new platforms. Since this hardware suite has supported previous launches, the GDS and operations team know that these components have passed the stress tests associated with supporting a launch; there are no unknowns by using the existing suite.

$\underline{\text { Reuse of personnel from project, operations and mission }}$ teams

Initially, the SMEX Project started with a large-scale army of subsystem developers, independent of each other, only meeting during reviews to consolidate interfaces and specific work issues. This was the approach used during the SAMPEX mission.

The SMEX Project then migrated to a combined System Implementation Team (SIT) approach that coordinated GDS development but was still independent and lagged behind the project spacecraft integration. The SWAS and FAST missions used this combined SIT approach. This approach helped to minimize the problems encountered during earlier GSFC programs, but did not completely solve the problems related to mission development and interfacing with the project and science teams.

Eventually, the SMEX Project (for the TRACE and WIRE missions) evolved into an Integrated Product Development Team (IPDT) that coordinated the development, project, and test groups. This IPDT took the form of the mission team (MT) and provided a sub-group related to mission readiness testing (MRT). The mission team also provided support to the project for meetings and reviews. The TRACE mission was first to use an IPDT [2]. The concept of an IPDT is not a new idea. However, the formation of the TRACE IPDT stressed joint development and partnering between previously independent development groups, breaking through traditional organizational barriers. A very significant portion of the cost to the previous mission was spent on communication via thick, formal documents. The 
TRACE IPDT minimized these costs by removing the formal barriers that required the generation of the documentation and concentrating on personal interaction and the use of the Internet as a repository for information sharing. Additionally, the flight operations team had ready access to all documentation regardless of where a particular operational workstation or laptop was located. By combining the workforce into the IPDT, any potential cost or schedule problems were mitigated because the standard organizational barriers were broken. By overcoming organizational barriers, the IPDT was able to define and own the process that allowed the TRACE and WIRE missions to reduce or eliminate duplication of effort. This same approach is currently in place on Triana.

As with the last two SMEX missions, the Triana mission works to minimize the differences between project personnel and GDS development and operations personnel. GDS and operations teams have supported the project reviews and weekly status briefings; operations personnel have also provided pre-launch spacecraft integration testing. The GDS and operations team used this information exchange to learn the nominal spacecraft operation concepts. By supporting the Project during reviews and weekly discussions, the GDS and operations teams have reduced development time and costs to meet the 3-year mission selection to launch criteria implicit in the SMEX and Triana charters.

While a major risk mitigation technique is to reuse the mission personnel and GDS HW and SW components, this is not to say that people do not move on to other programs or career opportunities. Nor does it mean that software systems used in supporting the first SMEX mission, SAMPEX, are being prepared for use on Triana. On the contrary, no software used for the SAMPEX launch in 1992 will be used for Triana. In fact, SAMPEX has undergone a "technological upgrade" benefiting from the improvements made during the course of the SMEX program and was retrofitted while on-orbit and collecting valuable science data. Likewise, a core group of engineers remain on Triana that were associated with SAMPEX but a significant number of personnel have moved onto other projects.

Bringing new team members "up to speed" on the next mission has been smooth and very successful because all documentation and review materials can be found on-line. This allows the new personnel to readily review the mission requirements, concepts, and processes.

Because the SMEX project was a series of similar spacecraft, once on-orbit the spacecraft were available for limited use in testing automation concepts and the on-orbit mission was used to train future operations staff. In fact, the TRACE and WIRE missions paved the way for the current autonomous operations concepts and allowed the formation of a standard $8 \times 5$ operations group. While the Triana spacecraft will be constantly in view of a ground station and supply a 24-hour live feed to the Triana control center; the
Triana operations team only will provide normal operations support during the standard day shift. However, during the launch and checkout phase, the control center will be staffed to provide $24 \times 7$ support for commanding and monitoring of the spacecraft instrument and subsystems.

In addition to the on-orbit spacecraft providing the basis for autonomous operation concepts, the operations personnel can be trained on these missions because of the similar/identical systems that will be used on the next generation mission. The experience and confidence gained is a significant risk mitigation technique for the operations team of the follow-on mission.

\section{CONCLUSIONS}

Although the first SMEX was launched on schedule in July 1992, the remaining spacecraft experienced significant delays due to launch vehicle problems. FAST was finally launched in August 1996. Because of these launch delays and the change to an ITOS-based architecture for mission set 2, it was decided in the fall of 1997 to re-engineer the SWAS GDS with the TRACE/WIRE approach. As a result the SMEX GDS mission team was supporting three missions simultaneously in different stages of development. It was a challenging task for the GDS mission team. However team members recognized the benefits of operating missions with the ITOS architecture. Figure 4 illustrates the revised schedules and subsequent launches of these three satellites.

Subsequently, the TRACE, SWAS and WIRE spacecraft were all launched within an 11-month interval. TRACE in April 1998, SWAS in December 1998 and WIRE in March 1999. The GDS and operations team performed extremely well for all the launches and on-orbit operations. Although WIRE experienced a severe on-board anomaly, scientific studies were performed using its sensitive star tracker. Today, 8 years after the first SMEX launch, all five spacecraft are operating and returning scientific and engineering data with the GDS achieving a cumulative data return of nearly $99 \%$.

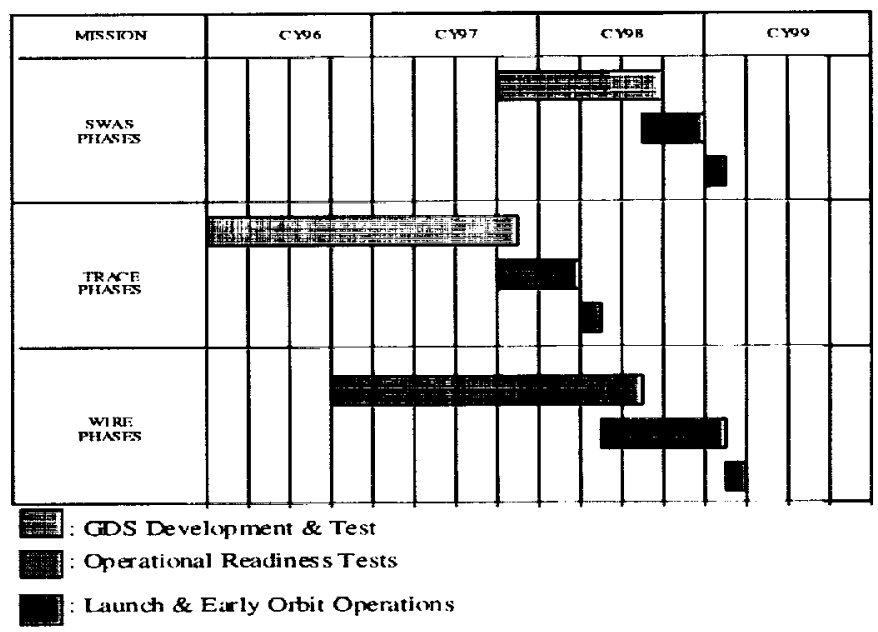

Figure 4. TRACE, SWAS \& WIRE Schedules \& Launches 


\section{REFERENCES}

[1] Paula Cleggett-Haleim, "NASA Selects Small Explorer Missions", NASA Headquarters Release 89-43, April 4, 1989.

[2] Scott Green, Dan Mandl, Keith Walyus, "Lights-Out Operations for the Transition Region and Coronal Explorer (TRACE) using Operational and Architectural Approaches," SpaceOps98 Conference, July, 1998.

[3] Joan Frank and Caroline Shulman, TRACE Innovations Continuous Improvement of the Testing Paradigm, CNMOS Presentation, February 11, 1998.

\section{BIOGRAPHIES}

John Catena is the Ground System Project Manager (GSPM) of the Triana mission at NASA GSFC since 1998.

Previously he was the GSPM for the first five SMEX missions starting in 1989 and completing that assignment in 1999.

Ralph Casasanta has been involved in ground system engineering for the SMEX missions since 1994. Over the course of his 24-year career with Computer Science Corporation, he has been involved in software development, test and systems engineering projects for NASA and the FAA.

Rick Saylor has been a member of the SMEX flight operations team at GSFC since 1994. A 6-year employee of Honeywell Technical Solutions, Inc., he has been involved in SMEX spacecraft integration, test and launch operations for the FAST and TRACE missions. He currently leads the flight operations contingent responsible for both of these activities on the Triana Project.

Craig Weikel is the lead ground systems engineer for the Triana mission. He has been a member of the GDS engineering team since the FAST mission. Previously he was a member of the software engineering team for SAMPEX. He has previously performed software development, test and system engineering duties for five earlier missions at GSFC in his 27-year career as a Computer Science Corporation employee. 